\title{
Treatment options for iatrogenic bile duct injuries with the loss of confluence
}

APASL Single Topic Conference Delta Hepatitis

June 27-28, 2019 Baku, Azerbaijan

Asadova A.A. ${ }^{1}$

${ }^{1}$ Azerbaijan Medical University. Departement of II surgical diseases. Baku, Azerbaijan.

Introduction. Iatrogenic bile duct injuries (IBDI) with the loss of confluence are the most feared types of biliary injury and represent $4 \%$ of all IBDI. The loss of confluence understood as when the right and the left hepatic ducts lose continuity with the common bile duct tree and to restore this continuity is a serious surgical challenge. Aim. The aim of this study is to share our results concerning the surgical treatment options of IBDI with the loss of confluence. Material and methods. During in a 10 years period (20082018) 105 patients with IBDI were admitted to our centers for surgical treatment. Among these patients there were only 13 patients with the loss of confluence (Strasberg E4 type). The diagnosis was confirmed by magnetic resonance cholangiopancreatography (MRCP) and contrast-enhanced computed tomography (CT) and classified according Strasberg classification as a Strasberg E4 type. Medical records of these patients were retrospectively analyzed and the general data were collected including the type of surgical procedures and postoperative outcomes. Results. Totally six males (46\%) and seven females (54\%) enrolled to our study (age ranged 23 to 66). The treatment options for these patients were following: nine patient (70\%) treated with double-barrel hepaticojejunustomy (HYS) and five of them were placed transhepatic transanastomotic drains. In two patient (15\%) were constructed neo-confluence. Finally in two patients(15\%) were performed portoenterostomy (Kasai procedure). In all patients Roux-en Y HYS was used. The postoperative complications were seen only in two patients. One patient experienced intraabdominal abcess and in one patient has been noted wound infection. Both cases were successfully managed with drainage and antibiotics. The postoperative mortality was nil. Conclusion. The treatment of Strasberg E4 type bilary injuries represents great technical difficulties. There are several surgical options (neo-confluence, double-barrel anastomosis, portoenterostomy) but, it is considered that double-barrel Roux-en $\mathrm{Y}$ HYS is the best choice for this patients. Key words. Biliary injuries, loss of confluence, neo-confluence, double-barrel anastomosis, portoenterostomy.

\footnotetext{
"Published on behalf of the Azerbaijan Gastroenterology and Hepatology Association for the APASL STC Hepatitis Delta. All rights reserved. (C) The Author 2019. For permissions please email: editor@ejcs.org or uptodate.az@gmail.com
} 